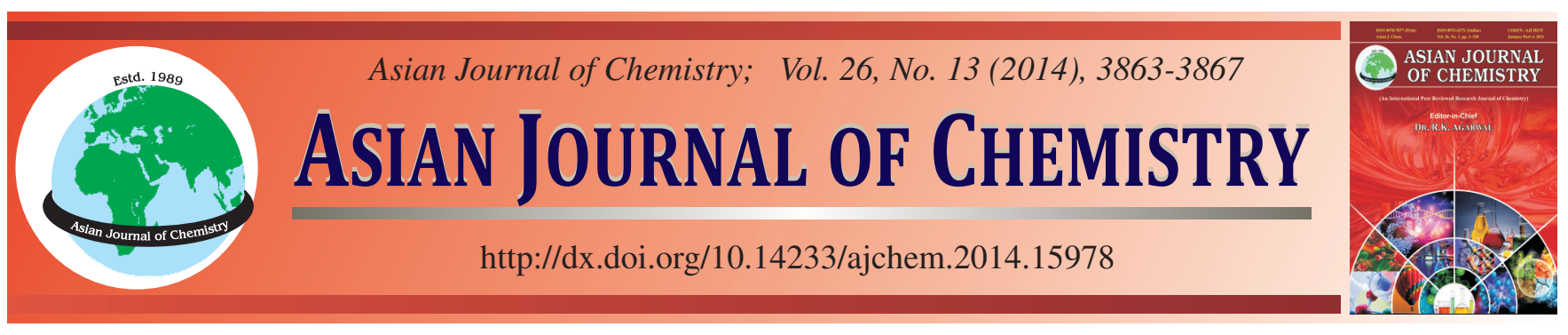

\title{
Analysis of Chemical Constituents of Volatiles and Petroleum Ether Extract from the Branch of Zanthoxylum bungeanum by GC-MS
}

\author{
Yuan-Yuan Liu ${ }^{\dagger}, \mathrm{XIN}_{\mathrm{N}} \mathrm{Shen}^{\dagger}$, Ya Zhang, Wei Cao*, Kai Wang, Shou-Zhu Xu and Si-Wang Wang*
}

Department of Natural Medicine, School of Pharmacy, Fourth Military Medical University, Xi'an 710032, P.R. China

*Corresponding authors: Fax: +86 29 83224790; Tel: +86 29 84773752; E-mail: siwangw@fmmu.edu.cn; caowei@fmmu.edu.cn

$\dagger$ These authors contributed equally to this work

\begin{abstract}
The essential volatiles were extracted by water steam distillation and the petroleum ether extract was extracted with alcohol and then petroleum ether from the branch of Zanthoxylum bungeanum. The chemical constituents of essential volatiles and petroleum ether extract were analyzed by GC-MS and their relative contents were calculated by area normalization method. 45 peaks were obtained and 34 components were identified from the volatiles and the major components were terpenes, alcohols and fats. As for petroleum ether extract, 60 peaks were obtained and 50 components were identified and the major components were organic acids, esters and alkanes.
\end{abstract}

Keywords: Zanthoxylum bungeanum, Volatiles, Petroleum ether, GC-MS.

\section{INTRODUCTION}

The genus Zanthoxy lum L. consists of about 250 species $^{1}$, which are mainly distributed in tropical and subtropical areas situated in Asia, America, Africa and Oceania ${ }^{2-6}$. In China, 45 species and 13 varieties are mainly distributed in south of the Yangtze river and southwest Provinces. Among them, 18 species are used as traditional Chinese medicine. The fruits, roots, branches, leaves of Zanthoxy lum L. are all medicine, which has been widely used for analgesia, anesthesia, antibacterial, pesticides and anti-tumor ${ }^{7,8}$. The plants of this genus are well known for containing several types of compounds, including volatile oil, alkaloids, amides, lignans, coumarins, fatty acids, flavones, terpenes, sterols and hydrocarbons ${ }^{9-13}$. Although many studies have shown the chemical constituents of Zanthoxy lum L extracted from the fruits or roots, there is little research on the compositions of branches. So the purpose of this study was to identify these compounds in the branches of Zanthoxy lum L. The gas chromatography-mass spectrometry (GC-MS) and computer spectrum search technique were used to identify the chemical composition of Zanthoxylum bungeanum. This study can provide test basis for the rational development and utilization of Zanthoxylum resources.

\section{EXPERIMENTAL}

The branches of Zanthoxylum bungeanum were collected from Hancheng city in the northwest of Shaanxi province in
September, 2011 in China. The fresh branches were dried in the shade at room temperature.

Sample preparation: Dried branches of Zanthoxylum bungeanum were cut into pieces. The volatile was extracted from the branches of Zanthoxylum $(0.75 \mathrm{~kg})$ by water steam distillation, then dried to pale yellow liquid using $\mathrm{Na}_{2} \mathrm{SO}_{4} .1 \mathrm{~mL}$ volatile was diluted to $5 \mathrm{~mL}$ with ethanol. Furthermore, the branches of Zanthoxylum $(0.75 \mathrm{~kg})$ were extracted with alcohol and then petroleum ether to get the petroleum ether extract. $20 \mathrm{mg}$ of petroleum ether extract was dissolved in $10 \mathrm{~mL}$ of petroleum ether. All the solutions were filtered through a $0.45 \mu \mathrm{m}$ filter.

Apparatus and GC-MS condition: The compositions were analyzed by an ISQ 110953 GC-MS system (Thermo Fisher Corp, USA). The condition was shown in the Table-1. Retention times were utilized as primary criterion for the peaks identification. Using the mass spectrometer as chromatographic detector offered additional data (P/N: 274, 102, 74 Thermo Data system) for the identification of the separated compounds. The identification method was reference standard compounds and compared the compositions isolated with the recorded spectra in MS library.

\section{RESULTS AND DISCUSSION}

Total gas chromatograms of volatiles and petroleum ether extract from branches of Zanthoxylum bungeanum were shown in Figs. 1 and 2. The relative contents of chemical constituents 
TABLE-1

\section{SELECTED PARAMETERS OF GC-MS CONDITION}

\begin{tabular}{|c|c|c|}
\hline Parameter & & Description \\
\hline Capillary column & \multicolumn{2}{|c|}{ GSBP-5MS ( $30 \mathrm{~m} \times 0.32 \mathrm{~mm}, 0.32 \mu \mathrm{m}$ film, $0.25 \mu \mathrm{m}$ ), poly ( $\%$ diphenyl, $95 \%$ dimethyl siloxane). } \\
\hline Carrier gas & \multicolumn{2}{|c|}{ Helium } \\
\hline Injection mode & \multicolumn{2}{|l|}{ Split } \\
\hline Column flow & A: $2.1 \mathrm{~mL} / \mathrm{min}$ & B: $1.4 \mathrm{~mL} / \mathrm{min}$ \\
\hline Split ratio & A: $50: 1$ & B: $10: 1$ \\
\hline Injection temperature & \multicolumn{2}{|c|}{$250{ }^{\circ} \mathrm{C}$} \\
\hline Oven temperature progarm & \multicolumn{2}{|c|}{$\begin{array}{l}\text { A: Initial temperature was } 80^{\circ} \mathrm{C} \text { (hold time } 1 \mathrm{~min} \text { ) then ramped at } 10^{\circ} \mathrm{C} / \mathrm{min} \text { to } 280{ }^{\circ} \mathrm{C} \text { (hold time } 2 \mathrm{~min} \text { ). } \\
\mathrm{B} \text { : Initial temperature was } 80^{\circ} \mathrm{C} \text { then ramped at } 3{ }^{\circ} \mathrm{C} / \mathrm{min} \text { to } 260^{\circ} \mathrm{C} \text { (hold time } 3 \mathrm{~min} \text { ), Again with ramped at } \\
20^{\circ} \mathrm{C} / \mathrm{min} \text { to } 2800^{\circ} \mathrm{C} \text { (hold time } 3 \mathrm{~min} \text { ). }\end{array}$} \\
\hline Ionization energy & $70 \mathrm{eV}$ & \\
\hline Scan range & A: $20-800 \mathrm{amu}$ & B: $35-400 \mathrm{amu}$ \\
\hline Event time & A: $0.5 \mathrm{~s}$ & B: $0.2 \mathrm{~s}$ \\
\hline Ionization source temperature & A: $200{ }^{\circ} \mathrm{C}$ & B: $260^{\circ} \mathrm{C}$ \\
\hline Transmission line temperature & A: $250^{\circ} \mathrm{C}$ & B: $280{ }^{\circ} \mathrm{C}$ \\
\hline
\end{tabular}

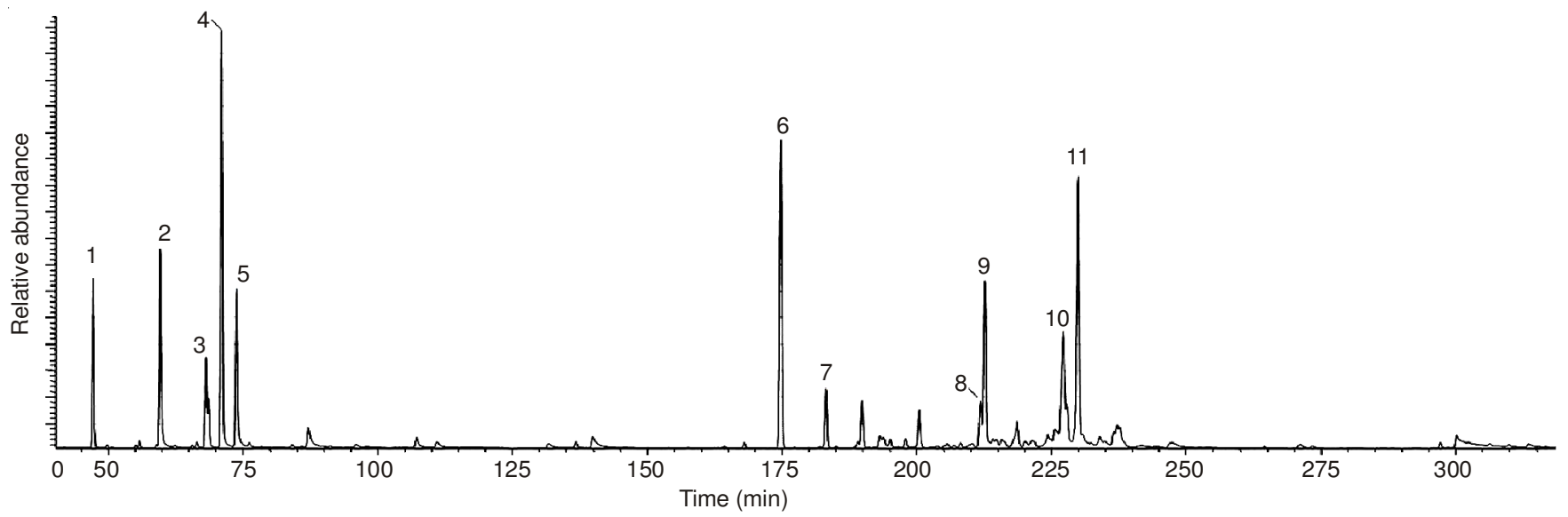

Fig. 1. GC-MS total ion chromatograms of volatiles; $1=\alpha$-pinene, $2=\beta$-myrcene, $3=\beta$-phellandrene, $4=\beta$-trans-ocimene, $5=\beta$-cis-ocimene, $6=$ caryophyllene, $7=\alpha$-caryophyllene, $8=(-)$-spathulenol, $9=(-)$-caryophyllene oxide, $10=$ torreyol, $11=\alpha$-cadinol

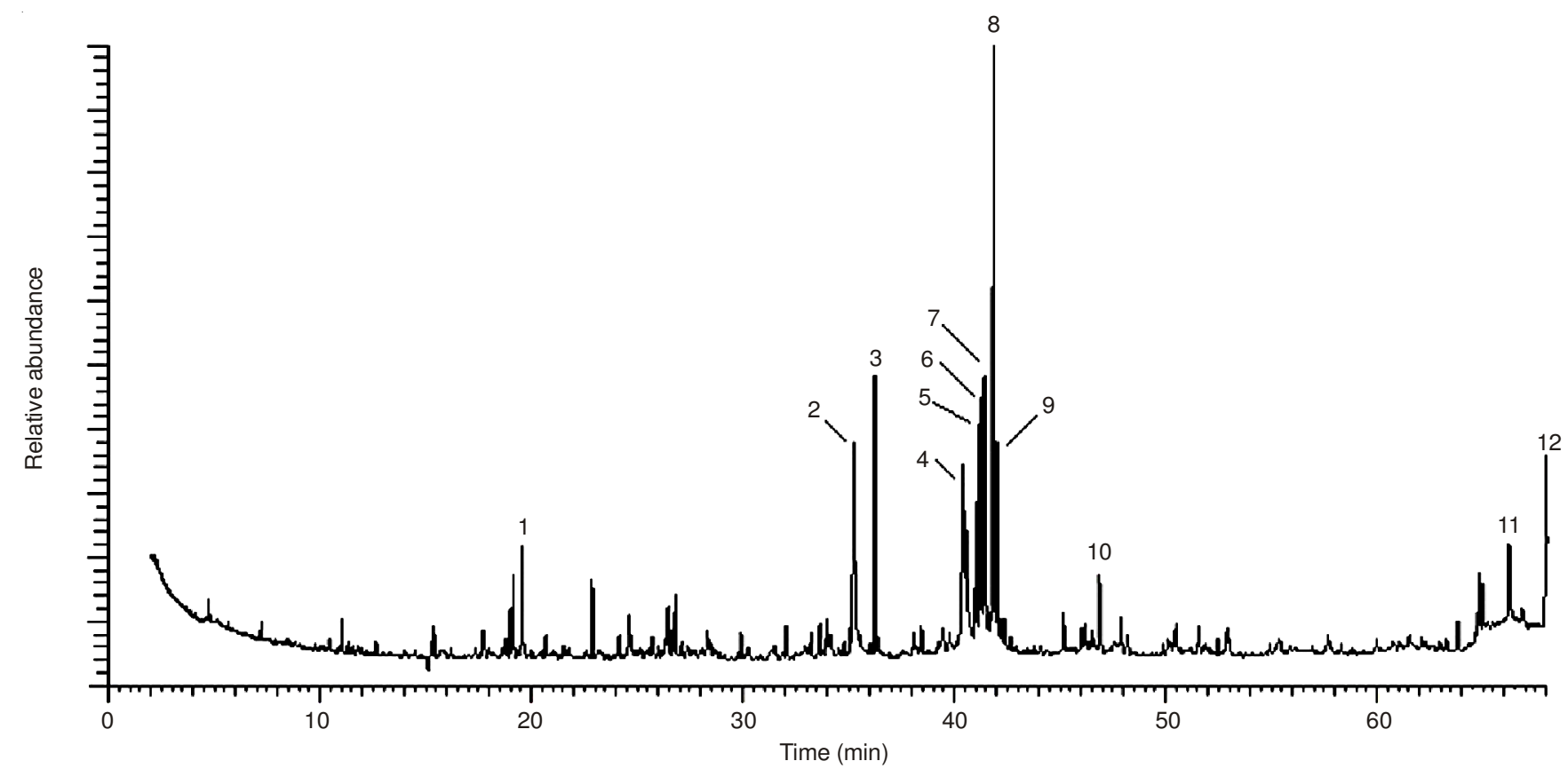

Fig. 2. GC-MS total ion chromatograms for petroleum ether extract part. $1=2,4$-di-tert-butylphenol, $2=n$-hexadecanoic acid, $3=$ hexadecanoic acid, ethyl ester, 4 = 2,3-dihydroxypropyl (9Z,12Z,15Z)-9,12,15-octadecatrienoate, $5=(3 \mathrm{Z}, 7 \mathrm{E}, 10 \mathrm{E})$-trideca-3,7,10,12-tetraenoic acid methyl ester, $6=$ mehyl 5,9-octadecadienoate, $7=$ cis-5,8,11,14,17-eicosapentaenoic acid, $8=$ doconexent, $9=\alpha$-cbz-L-arginine, $10=2,4,6$-tri-(tert-butyl)benzonitrile, 11 = campesterol, $12=\beta$-sitosterol 
TABLE-2

MASS DATA OF 36 COMPOUNDS IDENTIFIED FROM VOLATILES

\begin{tabular}{|c|c|c|c|c|c|}
\hline Peak No. & Compounds & $\mathrm{T}_{\mathrm{R}}(\min )$ & m.f. & m.w. & Area $(\%)$ \\
\hline 1 & $\alpha$-Pinene & 4.717 & $\mathrm{C}_{10} \mathrm{H}_{16}$ & 136 & 4.81 \\
\hline 2 & $\beta$-Pinene & 5.575 & $\mathrm{C}_{10} \mathrm{H}_{16}$ & 136 & 0.22 \\
\hline 3 & $\beta$-Myrcene & 5.985 & $\mathrm{C}_{10} \mathrm{H}_{16}$ & 136 & 6.74 \\
\hline 4 & Cymene & 6.642 & $\mathrm{C}_{10} \mathrm{H}_{14}$ & 134 & 0.18 \\
\hline 5 & $\beta$-Phellandrene & 6.808 & $\mathrm{C}_{10} \mathrm{H}_{16}$ & 136 & 2.91 \\
\hline 6 & 1,5-Cyclooctadiene & 6.858 & $\mathrm{C}_{10} \mathrm{H}_{16}$ & 136 & 1.61 \\
\hline 7 & $\beta$-trans-Ocimene & 7.092 & $\mathrm{C}_{10} \mathrm{H}_{16}$ & 136 & 14.4 \\
\hline 8 & $\beta$-cis-Ocimene & 7.375 & $\mathrm{C}_{10} \mathrm{H}_{16}$ & 136 & 5.98 \\
\hline 9 & $\gamma$-Terpinene & 7.608 & $\mathrm{C}_{10} \mathrm{H}_{16}$ & 136 & 0.18 \\
\hline 10 & Linalyl alcohol & 8.709 & $\mathrm{C}_{10} \mathrm{H}_{18}$ & 138 & 0.46 \\
\hline 11 & Terpinene-4-ol & 10.717 & $\mathrm{C}_{10} \mathrm{H}_{18}$ & 138 & 0.46 \\
\hline 12 & Acetic acid, bornyl ester & 13.675 & $\mathrm{C}_{12} \mathrm{H}_{20} \mathrm{O}_{2}$ & 196 & 0.24 \\
\hline 13 & Methyl nonyl ketone & 13.983 & $\mathrm{C}_{11} \mathrm{H}_{22}$ & 154 & 0.80 \\
\hline 14 & $(-)-\beta$-Elemene & 16.800 & $\mathrm{C}_{15} \mathrm{H}_{24}$ & 204 & 0.22 \\
\hline 15 & Caryophyllene & 17.475 & $\mathrm{C}_{15} \mathrm{H}_{24}$ & 204 & 12.4 \\
\hline 16 & $\alpha$-Caryophyllene & 18.308 & $\mathrm{C}_{15} \mathrm{H}_{24}$ & 204 & 3.28 \\
\hline 17 & $\gamma$-Muurolene & 18.892 & $\mathrm{C}_{15} \mathrm{H}_{24}$ & 204 & 0.20 \\
\hline 18 & Tridecan-2-one & 19.308 & $\mathrm{C}_{13} \mathrm{H}_{26}$ & 182 & 0.61 \\
\hline 19 & (-)- $\alpha$-Muurolene & 19.500 & $\mathrm{C}_{15} \mathrm{H}_{24}$ & 204 & 0.36 \\
\hline 20 & $\gamma$-Muurolene & 19.792 & $\mathrm{C}_{15} \mathrm{H}_{24}$ & 204 & 0.35 \\
\hline 21 & $\delta$-Cadinene & 20.042 & $\mathrm{C}_{15} \mathrm{H}_{24}$ & 204 & 1.55 \\
\hline 22 & Germacra-1(10),4,7(11)-triene & 20.808 & $\mathrm{C}_{15} \mathrm{H}_{24}$ & 204 & 0.17 \\
\hline 23 & (-)-Spathulenol & 21.175 & $\mathrm{C}_{15} \mathrm{H}_{24}$ & 204 & 2.07 \\
\hline 24 & (-)-Caryophyllene oxide & 21.258 & $\mathrm{C}_{15} \mathrm{H}_{24}$ & 204 & 7.51 \\
\hline 25 & (-)-Globulol & 21.408 & $\mathrm{C}_{15} \mathrm{H}_{26} \mathrm{O}$ & 222 & 0.41 \\
\hline 26 & Viridiflorol & 21.575 & $\mathrm{C}_{15} \mathrm{H}_{26} \mathrm{O}$ & 222 & 0.40 \\
\hline 27 & Bicyclo[2.2.2]oct-2-ene,1,2,3,6-tetramethyl & 21.633 & $\mathrm{C}_{12} \mathrm{H}_{20}$ & 164 & 0.21 \\
\hline 28 & (-)-Caryophyllene oxide & 21.858 & $\mathrm{C}_{15} \mathrm{H}_{24} \mathrm{O}$ & 220 & 1.64 \\
\hline 29 & 4,7-Octadecadiynoic acid, methyl ester & 22.008 & $\mathrm{C}_{19} \mathrm{H}_{30}$ & 360 & 0.36 \\
\hline 30 & Widdrol & 22.133 & $\mathrm{C}_{15} \mathrm{H}_{26} \mathrm{O}$ & 222 & 0.54 \\
\hline 31 & Torreyol & 22.717 & $\mathrm{C}_{15} \mathrm{H}_{26} \mathrm{O}$ & 222 & 6.69 \\
\hline 32 & $\alpha$-Copaene & 22.783 & $\mathrm{C}_{15} \mathrm{H}_{24}$ & 204 & 1.16 \\
\hline 33 & $\alpha$-Cadinol & 22.992 & $\mathrm{C}_{15} \mathrm{H}_{26} \mathrm{O}$ & 222 & 12.1 \\
\hline 34 & 4-Bromo-1-naphthylamine & 23.650 & $\mathrm{C}_{15} \mathrm{H}_{24} \mathrm{O}$ & 220 & 0.60 \\
\hline 35 & Sandaracopimaradiene & 29.717 & $\mathrm{C}_{20} \mathrm{H}_{32}$ & 272 & 0.24 \\
\hline 36 & Hexadecanoic acid & 30.017 & $\mathrm{C}_{16} \mathrm{H}_{32} \mathrm{O}_{2}$ & 256 & 0.60 \\
\hline
\end{tabular}

were calculated by area normalization method and the results were summarized in Tables 2 and 3.

Forty five peaks were obtained and 34 components were identified from the branches of Zanthoxylum by steam distillation, accounting for $95 \%$ of the total of the volatiles (Fig. 1). According to the percentage contents of comparison, the main constituents were $\beta$-trans-ocimene $(14.4 \%)$, caryophyllene (12.4\%), $\alpha$-cadinol (12.1\%), (-) -caryophyllene oxide $(7.51 \%), \beta$-myrcene $(6.74 \%)$ and torreyol $(6.69 \%)$. The major compositions of the petroleum ether were the terpenes, including 21 kinds of chemical constituents, accounting for $66.1 \%$ of the total compounds. The alcohols included 8 kinds of compositions, accounting for $23.2 \%$. Furthermore, the fats, ketones and silane acids were also detected in the volatiles (Table-2).

Sixty peaks were obtained and 50 components were identified from petroleum ether extract, accounting for $93.7 \%$ of the total petroleum ether extract (Fig. 2). According to the percentage content of comparison, the main constituents were doconexent (15.5\%), cis-5,8,11,14,17-eicosapentaenoic acid
(7.30\%), hexadecanoic acid, ethyl ester (6.95\%), methyl 5,9octad ecadienoate $(6.06 \%)$ and $n$-hexadecanoic acid $(5.84 \%)$. The major constituents of the petroleum ether extract were the organic acids, including 6 kinds of components, accounting for $34.5 \%$ of the total. The constituents of esters, including 11 kinds of components, accounting for $24.2 \%$. In addition, the petroleum ether extract also contained 18 kinds of alkanes, accounting for $21.3 \%$, ketones and phenols (Table-3).

\section{Conclusion}

Previous experiments have discovered the physiological activity of the volatile oil. Such as, cadinol has anti-diarrheal effect and can inhibit intestinal fluid accumulation of cholera toxin-induced mice ${ }^{14}$. Caryophyllene can modulate immune function by increasing NK cell activity in tumor-bearing mice ${ }^{15}$. Pinene has obviously contact toxicity to aphis gossypii glover. Ocimene and caryophyllene are used to synthesize the spices $^{16}$. All of these constituents of volatiles mentioned above can be extracted from the branches of Zanthoxylum bungeanum and have high contents. As for petroleum ether extract, this kind 
TABLE-3

MASS DATA OF 36 COMPOUNDS IDENTIFIED FROM PETROLEUM ETHER EXTRACT PART

\begin{tabular}{|c|c|c|c|c|c|}
\hline Peak No. & Compounds & $\mathrm{T}_{\mathrm{R}}(\min )$ & m.f. & m.w. & Area $(\%)$ \\
\hline 1 & Hexadecane & 11.02 & $\mathrm{C}_{16} \mathrm{H}_{34}$ & 226 & 0.71 \\
\hline 2 & 2-Butanone,1-[ (1,1-dimethylethyl)amino]-3,3-dimethyl & 15.23 & $\mathrm{C}_{10} \mathrm{H}_{21} \mathrm{NO}$ & 171 & 0.75 \\
\hline 3 & Tetradecane & 15.38 & $\mathrm{C}_{14} \mathrm{H}_{30}$ & 198 & 0.94 \\
\hline 4 & 2,6,10,15-Tetramethylheptadecane & 17.72 & $\mathrm{C}_{21} \mathrm{H}_{44}$ & 296 & 0.89 \\
\hline 5 & 11-Pentan-3-ylhenicosane & $\begin{array}{l}18.99 \\
26.67 \\
26.80\end{array}$ & $\mathrm{C}_{26} \mathrm{H}_{54}$ & 366 & 3.50 \\
\hline 7 & 2,4-Di-tert-butylphenol & 19.56 & $\mathrm{C}_{14} \mathrm{H}_{22} \mathrm{O}$ & 206 & 2.54 \\
\hline 8 & 5,14-Dibutyloctadecane & 22.86 & $\mathrm{C}_{26} \mathrm{H}_{54}$ & 366 & 1.94 \\
\hline 9 & 3-Ethyl-5-(2-ethylbutyl)octadecane & 24.15 & $\mathrm{C}_{26} \mathrm{H}_{54}$ & 366 & 0.69 \\
\hline 10 & Paromomycin & 24.62 & $\mathrm{C}_{15} \mathrm{H}_{26} \mathrm{O}$ & 222 & 1.16 \\
\hline 11 & 9-Hexylheptadecane & 25.73 & $\mathrm{C}_{23} \mathrm{H}_{48}$ & 324 & 0.66 \\
\hline 12 & Eicosane & 26.46 & $\mathrm{C}_{20} \mathrm{H}_{42}$ & 282 & 1.81 \\
\hline 13 & Heneicosane & $\begin{array}{l}28.32 \\
33.23\end{array}$ & $\mathrm{C}_{21} \mathrm{H}_{44}$ & 296 & 1.44 \\
\hline 14 & 5,8-Diethyldodecane & 29.92 & $\mathrm{C}_{16} \mathrm{H}_{34}$ & 226 & 0.69 \\
\hline 15 & 9-Hexylheptadecane & $\begin{array}{l}30.24 \\
42.37\end{array}$ & $\mathrm{C}_{23} \mathrm{H}_{48}$ & 324 & 1.14 \\
\hline 16 & 7,8-Epoxylanostan-11-ol, 3-acetoxy- & 31.39 & $\mathrm{C}_{32} \mathrm{H}_{54} \mathrm{O}_{4}$ & 502 & 0.55 \\
\hline 17 & Phthalicacid butyl undecyl ester & 32.04 & $\mathrm{C}_{23} \mathrm{H}_{36} \mathrm{O}_{4}$ & 376 & 0.96 \\
\hline 18 & 7,9-Di-tert-butyl-1-oxaspiro(4,5) deca-6,9-diene-2,8-Dione & 33.62 & $\mathrm{C}_{17} \mathrm{H}_{24} \mathrm{O}_{3}$ & 276 & 0.86 \\
\hline 19 & 2,4-Dimethylicosane & 33.97 & $\mathrm{C}_{22} \mathrm{H}_{46}$ & 310 & 0.97 \\
\hline 20 & 1,2-Benzenedicarboxylic acid, butyl octyl ester & 35.07 & $\mathrm{C}_{20} \mathrm{H}_{30} \mathrm{O}_{4}$ & 334 & 0.49 \\
\hline 21 & $n$-Hexadecanoic acid & 35.25 & $\mathrm{C}_{16} \mathrm{H}_{32} \mathrm{O}_{2}$ & 256 & 5.84 \\
\hline 22 & Ethyl 9-hexadecenoate & 35.53 & $\mathrm{C}_{18} \mathrm{H}_{34} \mathrm{O}_{2}$ & 282 & 0.30 \\
\hline 23 & Hexadecanoic acid, ethyl ester & 36.25 & $\mathrm{C}_{18} \mathrm{H}_{36} \mathrm{O}_{2}$ & 284 & 6.95 \\
\hline 24 & Erucic acid & 38.07 & $\mathrm{C}_{22} \mathrm{H}_{42} \mathrm{O}_{2}$ & 338 & 0.71 \\
\hline 25 & Ethyl 14-methyl-hexadecanoate & 38.44 & $\mathrm{C}_{19} \mathrm{H}_{38} \mathrm{O}_{2}$ & 298 & 0.81 \\
\hline 26 & 1,1-Didodecoxyhexadecane & 39.45 & $\mathrm{C}_{40} \mathrm{H}_{82} \mathrm{O}_{2}$ & 594 & 0.70 \\
\hline 27 & 1,3,5-Trimethyl-2-octadecylcyclohexane & 39.77 & $\mathrm{C}_{27} \mathrm{H}_{54}$ & 378 & 0.49 \\
\hline 30 & Methyl 5,9-octadecadienoate & 41.21 & $\mathrm{C}_{19} \mathrm{H}_{34} \mathrm{O}_{2}$ & 294 & 6.06 \\
\hline 31 & cis-5,8,11,14,17-Eicosapentaenoic acid & 41.40 & $\mathrm{C}_{20} \mathrm{H}_{30} \mathrm{O}_{2}$ & 302 & 7.30 \\
\hline 32 & Minaprine & 41.61 & $\mathrm{C}_{19} \mathrm{H}_{36} \mathrm{O}_{3}$ & 312 & 0.48 \\
\hline 33 & Doconexent & 41.85 & $\mathrm{C}_{22} \mathrm{H}_{32} \mathrm{O}_{2}$ & 328 & 15.5 \\
\hline 34 & Nalpha-cbz-L-arginine & 42.03 & $\mathrm{C}_{14} \mathrm{H}_{20} \mathrm{~N}_{4} \mathrm{O}_{4}$ & 308 & 4.78 \\
\hline 35 & Eicosanoic acid & 42.23 & $\mathrm{C}_{23} \mathrm{H}_{48}$ & 324 & 0.50 \\
\hline 36 & Pentacosane & 45.17 & $\mathrm{C}_{25} \mathrm{H}_{52}$ & 352 & 1.06 \\
\hline 37 & Ethyl iso-allocholate & 46.52 & $\mathrm{C}_{26} \mathrm{H}_{44} \mathrm{O}_{5}$ & 436 & 0.67 \\
\hline 38 & 2,4,6-Tri-(tert-butyl)benzonitrile & 46.86 & $\mathrm{C}_{19} \mathrm{H}_{29} \mathrm{~N}$ & 271 & 2.19 \\
\hline 49 & Heneicosane & 47.87 & $\mathrm{C}_{21} \mathrm{H}_{44}$ & 296 & 0.88 \\
\hline 40 & 9-Oximino-2,7-diethoxyfluorene & 48.19 & $\mathrm{C}_{17} \mathrm{H}_{17} \mathrm{NO}_{3}$ & 283 & 0.55 \\
\hline 41 & Tetratetracontane & 50.47 & $\mathrm{C}_{44} \mathrm{H}_{90}$ & 618 & 0.79 \\
\hline 42 & $\begin{array}{l}\text { 2-Butyryl-3-\{[2-(5-methoxy-2-methyl-1H-indol-3-yl)ethyl]amino }\}-2- \\
\text { cyclohexen-1-one }\end{array}$ & 51.55 & $\mathrm{C}_{22} \mathrm{H}_{28} \mathrm{~N}_{2} \mathrm{O}_{3}$ & 368 & 0.79 \\
\hline 43 & Docosanoic acid, ethyl ester & 52.89 & $\mathrm{C}_{24} \mathrm{H}_{48} \mathrm{O}_{2}$ & 368 & 0.59 \\
\hline 44 & Ethyl tetracosanoate & 57.66 & $\mathrm{C}_{26} \mathrm{H}_{52} \mathrm{O}_{2}$ & 396 & 0.54 \\
\hline 45 & $24 \alpha$-Methylcholesterol & 61.53 & $\mathrm{C}_{30} \mathrm{H}_{50} \mathrm{O}_{2}$ & 442 & 0.44 \\
\hline 46 & Stigmasta-5,22-dien-3 $\beta$-ol & 63.81 & $\mathrm{C}_{31} \mathrm{H}_{50} \mathrm{O}_{2}$ & 454 & 1.42 \\
\hline 47 & Sesamin & 64.80 & $\mathrm{C}_{20} \mathrm{H}_{18} \mathrm{O}_{6}$ & 354 & 1.55 \\
\hline 48 & 1,4-Bis(1,3-benzodioxole-5-yl)tetrahydro- $1 H, 3 H$-furo[3,4-c]furan & 64.97 & $\mathrm{C}_{20} \mathrm{H}_{18} \mathrm{O}_{6}$ & 354 & 1.22 \\
\hline 49 & Campesterol & 66.23 & $\mathrm{C}_{28} \mathrm{H}_{48} \mathrm{O}$ & 400 & 2.27 \\
\hline 50 & $\beta$-Sitosterol & 67.99 & $\mathrm{C}_{29} \mathrm{H}_{50} \mathrm{O}$ & 414 & 3.01 \\
\hline
\end{tabular}

of chemical constituent is often applied to chemical production. Such as, hexadecanoic acid ethyl ester is used in lubricant. Erucic acid is used to manufacture the artificial fiber ${ }^{17}$. The identification of the chemical constituents of volatiles and petroleum ether extract from the branch of Zanthoxylum bungeanu would contribute to making full use of herb resource.

\section{REFERENCES}

1. J. Hu, W.D. Zhang, Y.H. Shen, C. Zhang, L. Xu, R.-H. Liu, B. Wang and X.-K. Xu, Biochem. Syst. Ecol., 35, 114 (2007).

2. D. Geng, D.X. Li and Y. Shi, Chin. J. Nat. Med., 7, 274 (2009).

3. T. Hatano, K. Inada, T. Ogawa, H. Ito and T. Yoshida, Phytochemistry, 65, 2599 (2004). 
4. K. Hashimoto, K. Satoh, Y. Kase, A. Ishige, M. Kubo, H. Sasaki, S. Nishikawa, S. Kurosawa, K. Yakabi and T. Nakamura, Planta Med., 67, 179 (2001).

5. Quanbo Xiong, S. Dawen, H. Yamamoto and M. Mizuno, Phytochemistry, 46, 1123 (1997).

6. C.-T. Chang, S.-L. Doong, I.-L. Tsai and I.-S. Chen, Phytochemistry, 45, 1419 (1997).

7. S. Wine-show, T. Ian-Lih, T. Che-Ming and C. Ih-Sheng, Phytochemistry, 36, 213 (1994).

8. K. Jang, Y.H. Chang, D.-D. Kim, K.-B. Oh, U. Oh and J. Shin, Arch. Pharm. Res., 31, 569 (2008).

9. A.P.K. Nissanka, V. Karunaratne, B.M.R. Bandara, V. Kumar, T. Nakanishi, M. Nishi, A. Inada, L.M.V. Tillekeratne, D.S.A. Wijesundara and A.A.L. Gunatilaka, Phytochemistry, 56, 857 (2001).
10. I. Chen, T. Chen and W. Lin, Phytochemistry, 52, 357 (1999).

11. Y. Kashiwada, C. Ito, H. Katagiri, I. Mase, K. Komatsu, T. Namba and Y. Ikeshiro, Phytochemistry, 44, 1125 (1997).

12. S.L. da Silva, P.M.S. Figueredo and T. Yano, Pharm. Biol., 44, 657 (2006).

13. S.L. Da Silva, P.M.S. Figueiredo and T. Yano, Acta Amazon., 37, 281 (2007).

14. C.B. Duschatzky, A.N. Martinez, N.V. Almeida and S.L. Bonivardo, J. Essent. Oil Res., 16, 626 (2004).

15. T. Ashitani, A.K. Borg-Karlson, K. Fujita and S. Nagahama, Nat. Prod. Res., 22, 495 (2008).

16. W.N. Setzer, J. Essent. Oil Bear Pl., 10, 475 (2007).

17. P.P. Fu, S.-H. Cheng, L. Coop, Q. Xia, S.J. Culp, W.H. Tolleson, W.G. Wamer and P.C. Howard, J. Environ. Sci. Health C, 21, 165 (2003). 